\title{
The Position Design of Rail Transit Train Based on RFID-Geomagnetic Combined Positioning
}

\author{
Wenxiang LI*, Qinzhen HUANG \\ The four section of the first ring road \\ Southwest Minzu University \\ Chengdu, Sichuan, China \\ *Email: 944033818@qq.com \\ +* Corresponding author
}

\begin{abstract}
This paper presents a design based on RFID-terrestrial magnetism combined positioning which can achieve the accuracy and reliability of the rail transit train positioning, and introduces in detail.
\end{abstract}

Keywords-rail transit train; RFID; terrestrial magnetism; combined positioning

\section{INTRODUCTION}

With the rapid development of science and technology, urban rail transit has become the first choice for all countries to solve the urban traffic problems because of its safety, reliability, energy saving, environmental protection and high operation speed etc..Train positioning technology plays an important role in its operating system, which is directly related to the safety of the train operation [1]. Therefore, here a detailed description of a RFID-terrestrial magnetism combined positioning of the rail transit train positioning design, hereinafter referred to as position detector.

\section{PRINCIPLE OF POSITION DETECTOR}

The train position detector uses the combined detection principle of RFID and geomagnetic vehicle detection. And position detector consists of hardware circuit module, structural cavity module and software module.The hardware circuit module includes power supply module (power adapter, charging circuit board and lithium battery), RFID module (RFID antenna, RFID reader), geomagnetic detector module, $470 \mathrm{MHz}$ wireless module and MCU control module. The hardware circuit module is fitted into the structural cavity module to form the functions of RFID and geomagnetic sensor detection, MCU data fusion and data transmission. The software module contains signal processing and reception and transmission of data[2]. The principle diagram of the position detector is shown in Figure 1:

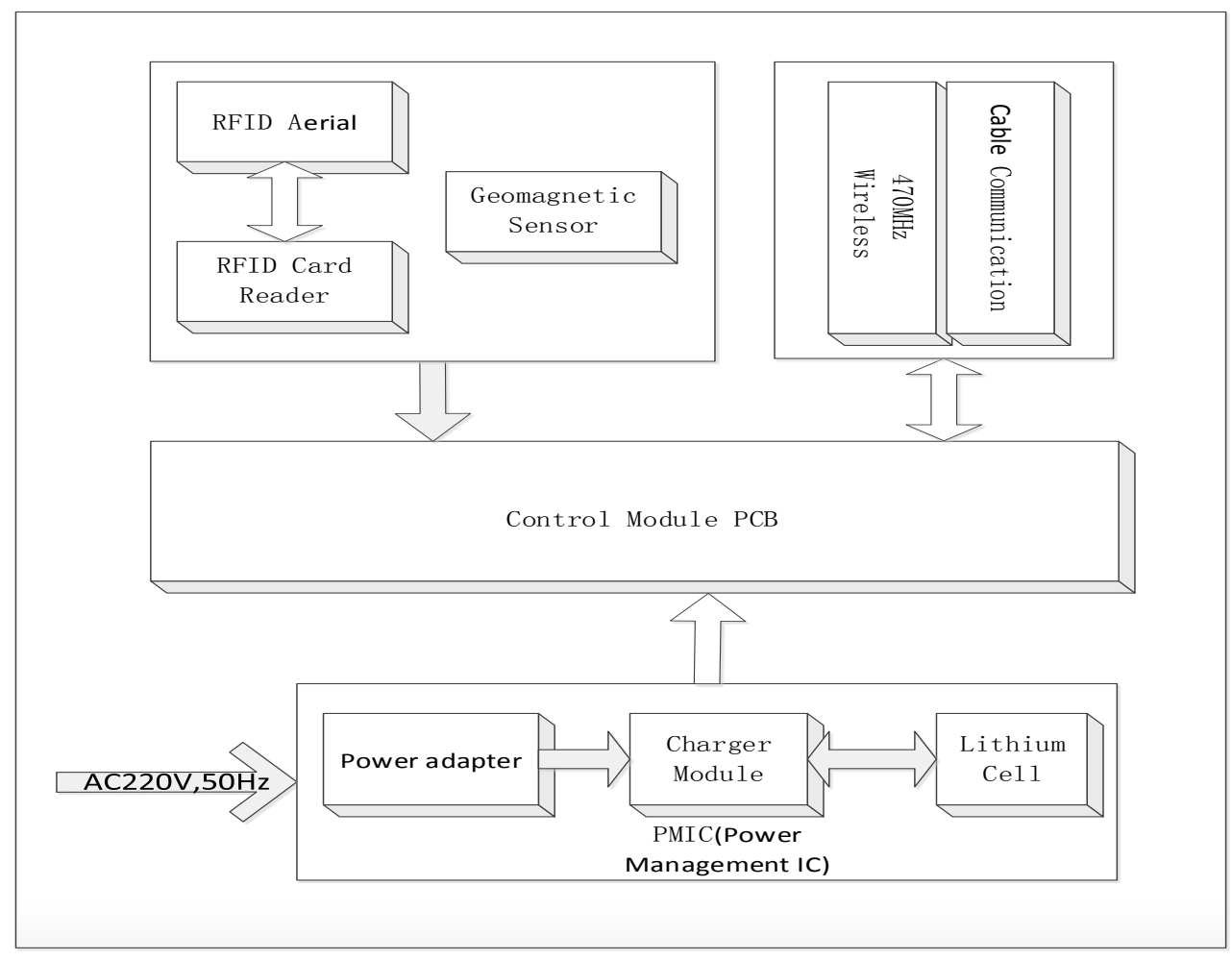

Figure 1. The diagram of position detector 


\section{DESIGN OF POSITION DETECTOR HARDWARE}

In the position detector, the RFID antenna detects the RFID tag on the train and then signals it through the RFID reader, and then sends the signal to the STM32F103RCT6 micro controller via the RS485 serial port.The geomagnetic sensor changes the magnetic field of the magnetic chip through the train to produce the signal, and then amplifies the signal through the OPA2320 and then inputs it to the AD port of the STM32F103RCT6 microcontroller. MCU to collect signal RFID and geomagnetic sensor after data processing, the processed data is transmitted to the roadside unit via $470 \mathrm{MHz}$ wireless or wired communication link RS485.

\section{A. $M C U$}

The design power supply using the mains power supply, the microcontroller does not have much power requirements.In the design, RFID and single-chip use of RS485 communication between the $470 \mathrm{MHz}$ wireless module and the use of SPI communication between the microcontroller, geomagnetism sensors and temperature sensors directly into the microcontroller's AD port, single-chip and roadside unit between the wired communication for RS485.

The main task of the project microcontroller STM32F103RCT6:

Receive signals from RFID and magnetoresistive sensors, then process them.

Communicate with the roadside unit via RS485 or $470 \mathrm{MHz}$ wireless transceiver module.

Time synchronization through the RTC function and roadside unit.

MCU gets the vehicle passing information by analyzing the data input by the RFID, detecting the magnetoresistive sensor input based on the A / D sampling value of the calculation and analysis of the state machine principle, comparing the baseline value, the current value, the peak value and a series of parameters.

\section{B. Geomagnetic Sensor}

The geomagnetic sensor is calculated by externally changing the magnetic field of the geomagnetic chip so that it can produce signals. This design uses HMC1022 geomagnetic sensor chip, and geomagnetic sensor in this design is the core detection mode.

The actual circuit is shown in Figure 2.

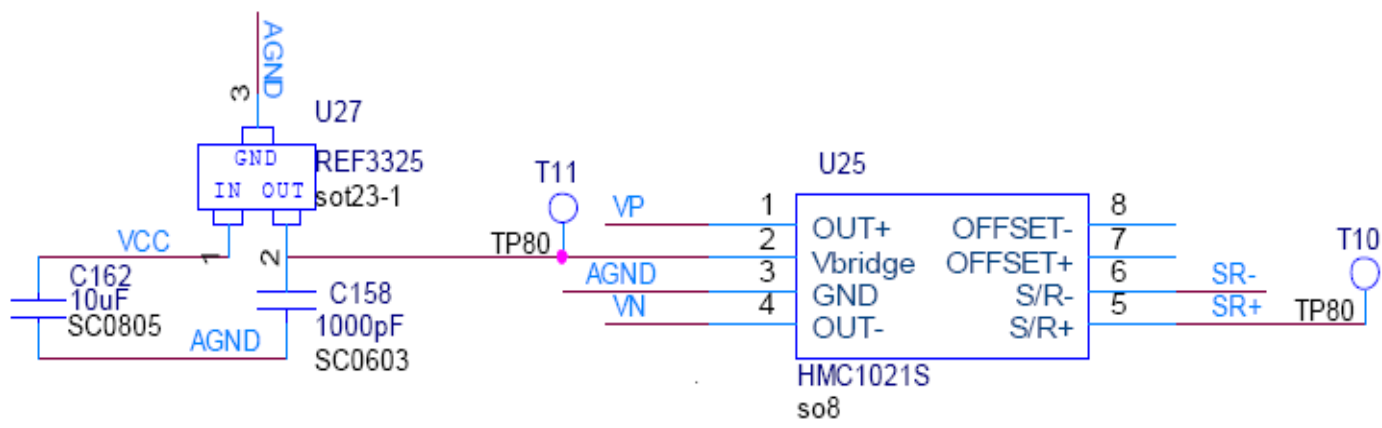

Figure 2. The schematic of the actual circuit

When the magnetoresistive sensor is disturbed by an external high magnetic field, such as permanent magnets, power lines and other environments. when the interference magnetic field is greater than 10-20Guass, it may lead to sensor magnetization. Sensor magnetization is not a permanent damage, it shows a sharp decrease in sensor sensitivity and an output viscosity. By setting / resetting, the sensor magnetization can be eliminated and the normal sensitivity state is restored [3]. The set / reset circuit of the module is shown in figure 3 : 


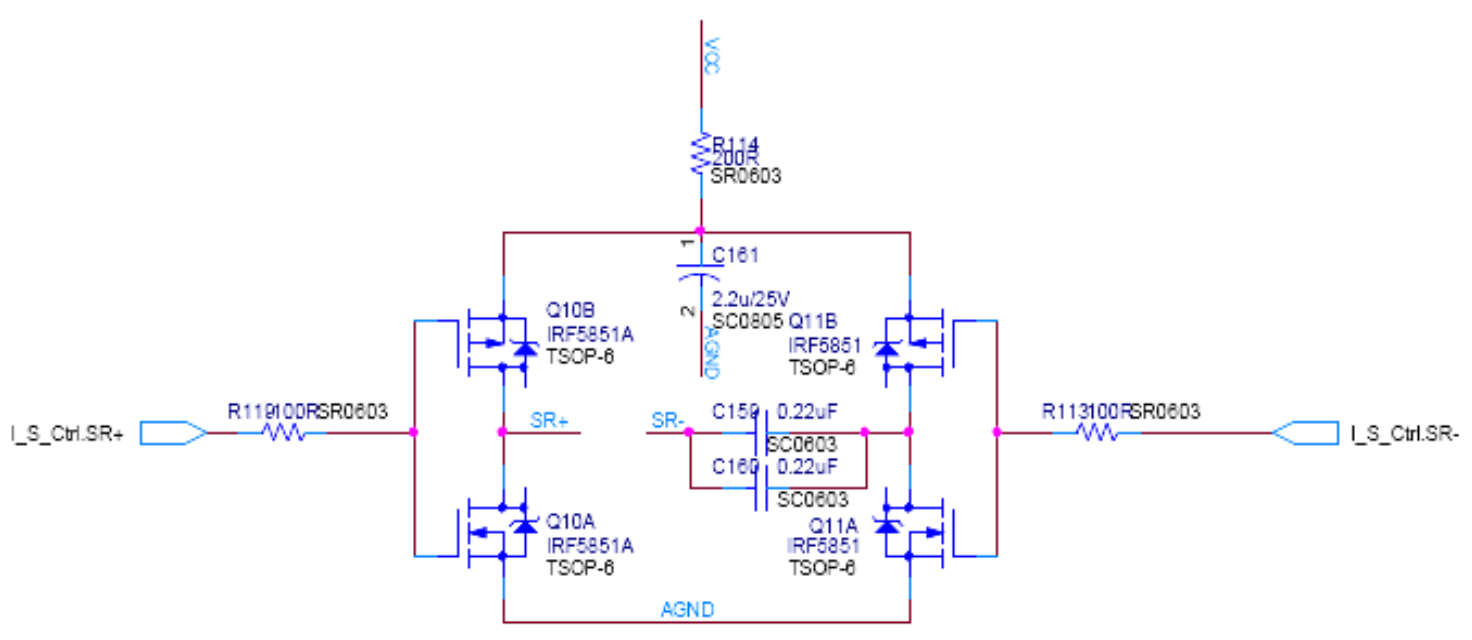

Figure 3. The set / reset circuit

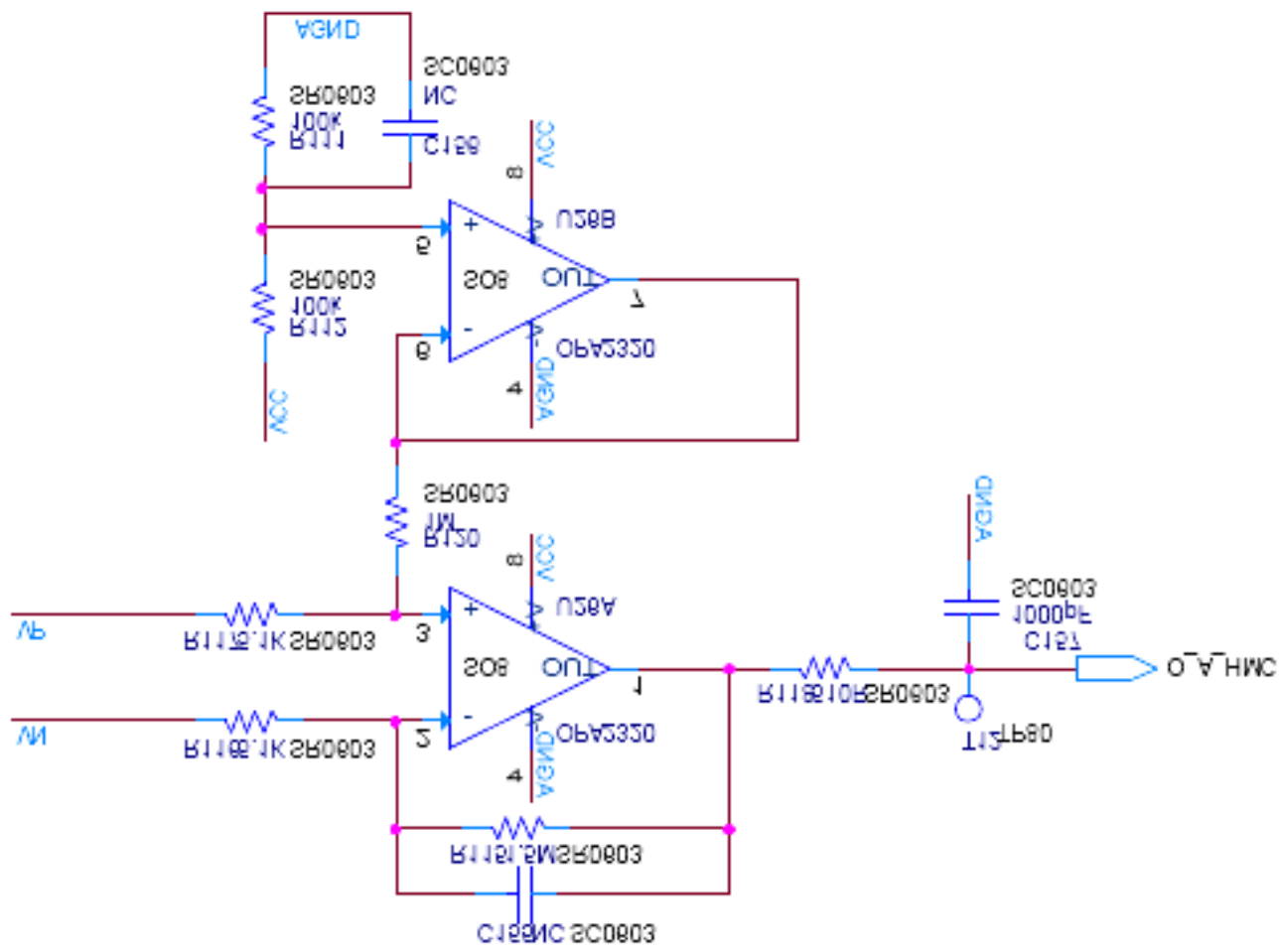

Figure 4. The schematic of the amplifier circuit

During the Ctrl_SR+ and Ctrl_SR- level jumpï1/1/4transi tioni $1 / 4 \%$ procedures, there will be two pulse currents whic $h$ are positive and negative on both ends of them. The imp ulse width is a few microns. The setting/resetting operation is completed after the pulse current is sent to the correspon ding S/R port of the sensor(transducer). 
The output voltage signal of magneto resistance sensor needs to be amplified and sent into the 12bitA/D of STM32F103RCT6 for sampling and quantizing. In this design, the OPA2320 operational amplifier is used, which has the maximum $65 \mathrm{uV}$ offset voltage, $12 \mathrm{~V} / \mathrm{uV}$ slew rate, and greater than $20 \mathrm{MHz}$ bandwidth. The schematic of the amplifier circuit is shown in figure 4.

In figure R1165/R1151, the theoretical magnification of the circuit is $M$, and $M$ is first theoretically estimated, and then through a large number of tests to verify its rationality and correction. Several factors must be considered in the selection of $M$ values:

The $\mathrm{M}$ value can not be too big or too small, too big can cause loss of output saturation and the ability of judgment can also lose, on the contrary, too small can cause effective points of the quantized signal reduce. So that it will directly reduce the algorithm for vehicle detection, threshold settings and detection of radius and other settings of the resolution;

The $M$ value set should widely collect the voltage output waveform when vehicles across the sensor in the same situation, focusing on the reference peak;

The $M$ value setting should refer to the STM32F103RCT6 A/D quantization strategy and threshold setting. The STM32F103RCT6 adopts 12bitA/D, and the designed supply voltage is VCC $3.3 \mathrm{~V}$, so the analog voltage input range is $0 \sim 3.3 \mathrm{~V}$. The design requires that the vehicle pass through the detection threshold at $20 \sim 100$.

HMC-1021S magnetic sensor sensitivity is $1 \mathrm{mV} / \mathrm{V} / \mathrm{Gauss}$ according to the typical device manual, according to the sensitivity formula of the excitation voltage in the case of $2.5 \mathrm{~V}$ magnetoresistive sensor output sensitivity is $2.5 \mathrm{mV} / \mathrm{Gauss}$, we set up a threshold for 20 100

The peak value of the magnetic field caused by the sensor when vehicle passes is tens to hundreds of mGauss, $50 \mathrm{mGauss}$, for example, assuming that the peak when the vehicle passes is detected. Then, the threshold is assumed to be 50 .

$$
\frac{2.5 \times 0.01 \times 0.05}{3.6} \times \mathrm{M} \times 4095=50
$$

The $\mathrm{M}$ is approximately 352 times from the previous model, and is considered as in the long run of actual testing and debugging, so the $\mathrm{M}$ value is 300 .

RFID is the abbreviation of Radio Frequency Identification, that is, radio frequency identification, commonly known as electronic tags. RFID is a simple wireless system, which is mainly used to control, detect and track objects. The RFID system consists of an interrogator (reader), a tag (transponder or RF card), and an antenna [4]. The tag consists of a coupling element and a chip.

The design of the RFID system in the air medium of the farthest detection distance can reach $15 \mathrm{~m}$, and detection speed can reach $80 \mathrm{Km} / \mathrm{h}$ in the car test. The detection distance and speed of the selected RFID system are beyond the requirements of the system design, which is because the environment used in the design is complex, and the attenuation of the field signal is great.
In this design, the detection distance of RFID is about $10 \mathrm{~m}$ because of the complex design of the structure, and after selecting, we choose the ultra-high frequency antenna UA1313, it is suitable for the RFID tag and the reader to detect the distance of about $15 \mathrm{~m}$.

\section{SOFTWARE DESIGN OF POSITION DETECTOR}

Software design refers to STM32F103RCT6 single-chip program, including the detection, acquisition, and data transmission. STM32F103RCT6 single-chip microcomputer through the acquisition of geomagnetic variation of data, detection of the data collected by the RFID, the logical operation of the data and comprehensive judgments, transmitted the results through the RS485 to the roadside unit; if the RS485 link breaks down, from the $470 \mathrm{MHz}$ wireless module the data is sent to the roadside unit.

The sensor detection part using the cycle detection work style, MCU completed the geomagnetic detection part of the $\mathrm{AD}$ sampling and logic operation in the detection period; RFID reader reads the tag information; operation and comprehensive judgment of data [5]. When the detection detects the vehicle information, MCU through the RS485 interface or through the I/O communication port and wireless RF transceiver module sends the information to the roadside units. Besides the testing period, MCU will regularly do the synchronization and communication verification operation with roadside units to ensure the unification of the system time and the fluency of the communication link.

Geomagnetic detection is one of the core design of the sensor, the average of environmental magnetic field value by MCU's AD sampling, namely the baseline value as a basis for judging the vehicle, when the vehicle through the top of the sensor can cause a relative to the baseline value of environmental magnetic field varying magnetic field perturbation, by sampling the instantaneous value of $\mathrm{AD}$ magnetic field, the instantaneous value (default 1 sampling points) and the baseline value of the deviation between the degree (denoted as the threshold, the default value is 35 ) to determine whether there is a car [6]. As shown in the diagram below, the baseline can be considered as a normal change within a small range (default is 4). Within this range, slow baseline changes are normal (baseline acquisition methods are described in detail later). When the difference between the magnetic field and the baseline exceeds the preset range (the default value is 35), it can be used as one of the criteria for vehicle detection. A car through the decision process of the sensor: the magnetic field from the near baseline $(+4)$ change exceeds the threshold (positive) - exceeds the threshold (negative) back to the magnetic field near the baseline $(+8$ and keep enough sampling points of continuous fall in the range, the default sampling point 10). The above is a vehicle which is passing through the magnetic field above the sensor and the software decision process. The changes of the magnetic field are shown in figure 5:

The sampling interval is $8 \mathrm{~ms}$, AD sampling, according to the deviation from the baseline to determine whether there are cars; from no car to car requires at least 1 points meet the car condition (deviation is greater than or equal to 35 compared with the baseline value); from cars to no car requires at least 10 consecutive a little to meet car free 
conditions (deviation less than or equal to 8 compared with the baseline value). The collective process of the baseline is shown below in figure 6 :

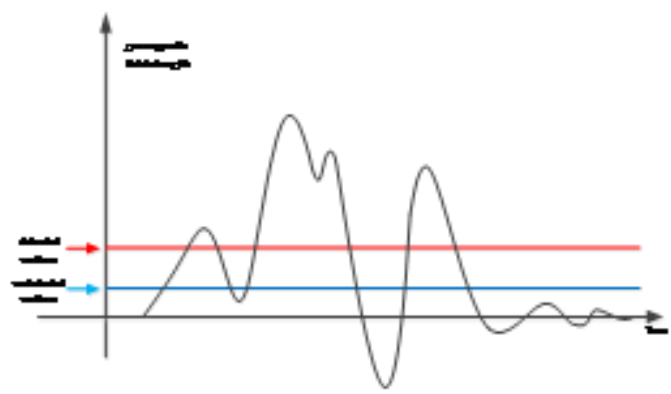

Figure 5. The changes of the magnetic field

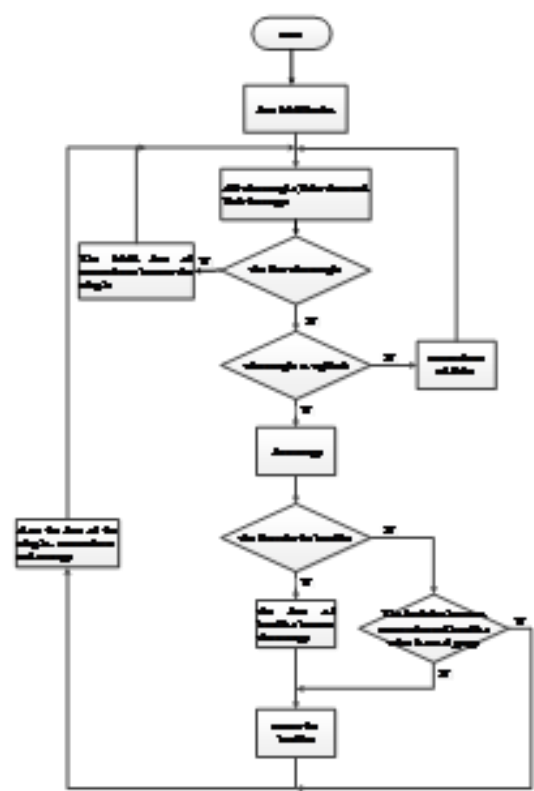

Figure 6. The collective process of the baseline

Baseline acquisition parameters include accumulators, sampling times, upper limit of sampling times, (default is 256), baseline count, mean, floating range of baseline (default is 4). After the initialization of parameters, limit the value of the collection scope; when the collection reached 256 times, calculate the average value; if it is the first time to obtain baseline values, the mean baseline value will be as the initial value; if the baseline value has been obtained, and the deviation of mean and baseline values is greater than 4 , then resample to obtain a new baseline value. If the deviation of mean and baseline is less than or equal to 4 , then update the baseline value and resample. In practice, baseline values change slowly.

\section{VERIFICATION BY EXPERIMENTS}

In order to verify the accuracy of position detector, $\mathrm{XX}$ group's road is chosen in the field testing; the position detector as shown is installed in the middle of the road, RFID tags installed on the car, the car back and forth through the position detector for 100 times. The result of the detection is shown in Table 1. Because of the use of composite detection, this design greatly improves the detection's accuracy; the accuracy rate can reach $99 \%$, which can meet the requirements of train positioning. This method can be applied to the rail transit train positioning function.

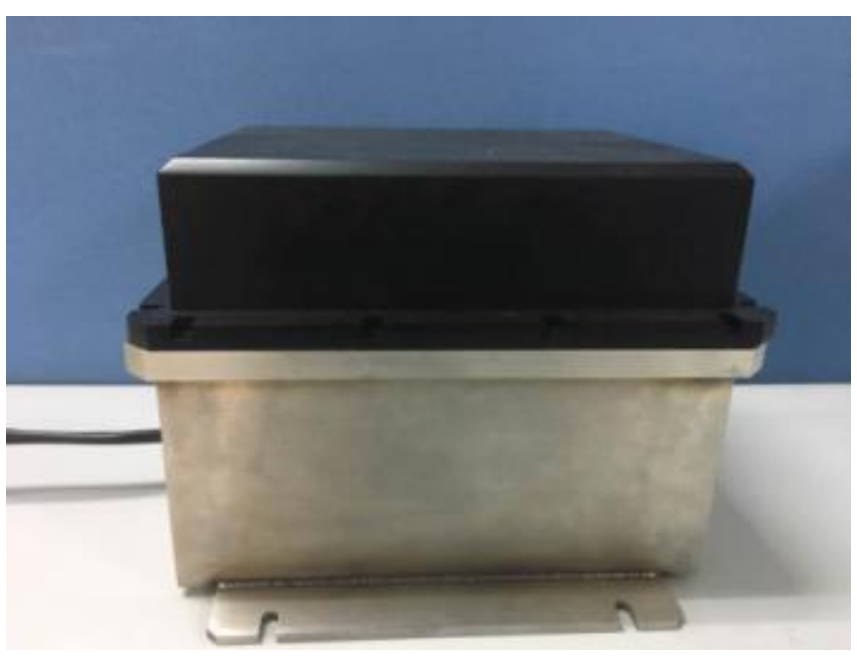

Figure 7. Physical map of position detector

TABLE. 1 THE DETECTION RESULT OF VEHICLE NUMBER

\begin{tabular}{|l|l|l|l|}
\hline Vehicle type & $\begin{array}{l}\text { Detection } \\
\text { value }\end{array}$ & Actual value & $\begin{array}{l}\text { Accuracy } \\
\text { rate }\end{array}$ \\
\hline The car & 100 cars & 99 cars & $99 \%$ \\
\hline
\end{tabular}

\section{CONCLUSION}

This paper realizes a rail transit train positioning system based on RFID-geomagnetic combination positioning, which can be applied to the positioning of rail transit train, and it can greatly improve the accuracy of positioning.In modern society, trams become the development trend of rail transit, the next step of the direction of the design can be applied to the tram positioning system, and study in detail.

\section{REFERENCES}

[1] The Systematic Lecture of Urban Rail Traffic Signal (NAM) [Z].

[2] Yang Zhikai. Research on vehicle detection sensors based on Geomagnetism technology. [D]. Hangzhou: Hangzhou Dianzi University, 2013.

[3] Pei Yi, Yu south, Liu Qi et al. Anisotropic magnetoresistive sensor principle and application J. Instrument technique and sensor, 2004, (8): $26-27$.

[4] Zhen Yan, Li Xiangzhen. Research and application of.RFID technology [J]. Digital communications, 2011, 38 (1): 32-35.

[5] history. Research on traffic state acquisition technology based on information sensor [D]. Hangzhou: Hangzhou Dianzi University, 2013.

[6] Yang Huifeng, Zhou Qi. A new type of vehicle detector based on single chip microcomputer and magnetoresistive sensor [J]. Journal of Chongqing University of Technology (NATURAL SCIENCE) 